\title{
Reducing Design Risk for a Nearshore Breakwater in the Orkney Islands
}

\author{
Daniel Rodger and Josh Harris
}

\begin{abstract}
This paper presents a case study of a conceptual breakwater design to address wave overtopping experienced at the Churchill Barrier No. 2, in the Orkney Islands, UK.

Four approaches to size armourstone have been investigated to account for uncertainty within nearshore wave estimates. A deterministic design approach combined with a joint probability analysis resulted in a stable rock armour weight of 9 tonnes. The use of an ultimate limit state based the unlikely scenario of a 200 -year wave, water level and $95^{\text {th }}$ percentile climate change estimate occurring simultaneously resulted in rock armour of 13 tonnes. The use of a probabilistic design incorporating partial safety factors resulted in rock armour of 20 tonnes. The use of Monte Carlo approach indicated an armour weight of 12 tonnes would capture $95 \%$ of the uncertainty within simulated 200-year extreme events, which is supporting the ultimate limit state calculations.
\end{abstract}

Index Terms-Breakwater, probabilistic design, rock armour.

\section{INTRODUCTION}

Breakwaters are constructed to provide protection to coasts, ports and harbours from waves, and to manage sediment on the beach. For over 100 years rubble mound breakwaters have been constructed worldwide, designed using various types of armourstone, of different shapes and sizes. Since the 1930s the design methodology to calculate the size of armour units has changed very little, with coastal engineers relying on empirical formulae based on estimates of extreme wave conditions in front of a structure.

A rubble mound breakwater has been considered to address wave overtopping experienced at the Churchill Barrier No. 2, in the Orkney Islands, UK. The series of causeways connecting Kirkwall to South Ronaldsay in the Orkney Islands are known as the Churchill Barriers, and are now used as a road access between the north and south islands. Wave overtopping of the barriers occurs during storm events that originate in the North Sea with waves propagating into Holm Sound to break against the eastern side of Barrier No. 2 (refer to Fig. 1). In a response to the threat to public safety the construction of a new breakwater has been investigated to reduce the overtopping experienced during extreme events to within tolerable limits.

The breakwater has been designed using the numerical model SWAN (Simulating WAves Nearshore) to optimise performance and reduce construction costs. Nearshore wave conditions from the SWAN model were used to design the geometry of the breakwater which used standard

Manuscript received December 20, 2013; revised March 7, 2014. This work was supported by the Orkney Islands Council.

The authors are with JBA Consulting, Broughton Hall, Skipton, UK (e-mail: danielrodger@jbaconsulting.com,joshharris@jbaconsulting.com). methodologies to design the armourstone frontage. While the use of the numerical model has increased confidence in the calculation of extreme waves at the proposed structure, there remains additional uncertainty due to; offshore extreme wave calculations, surge estimates, climate change projections, the numerical solution method and the accuracy of the numerical model. Three standard methods have been investigated to account for the inherent uncertainty within armourstone calculations and to mitigate the risk of undersizing armour. These have included the use of deterministic design formulae combined with joint probability analysis of marginal extreme wave and water level conditions, the use of an ultimate limit state based on the improbable scenario of a 200-year wave, water level and peak climate change estimate occurring simultaneously, and the use a probabilistic design (in addition to the joint probability assessment) incorporating partial safety factors published in the Coastal Engineering Manual (CEM) [1]. To refine the armourstone calculations, ensemble wave modelling was undertaken using a simplified Monte Carlo approach where 500 different extreme 200-year events were simulated, incorporating various levels of uncertainty within extreme wave, water level and climate change estimates.

The following sections describe the use of numerical modelling to support the design process and the approaches to improve the certainty in armourstone calculations.

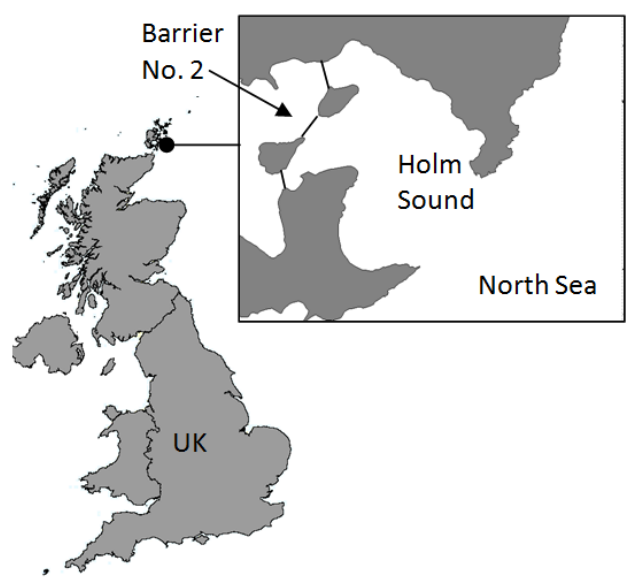

Fig. 1. Study site within Orkney Islands, UK.

\section{Conceptual Design}

The conceptual design of the proposed breakwater has been supported by numerical modelling software. Storm scenarios were simulated using the SWAN model and setup through the Delft3D-WAVE modelling shell. SWAN is a third generation wave transformation model incorporating complex physics for the description of nearshore wave transformation processes. 
The wave model domain has extended approximately ten kilometres east of the Orkney Islands. A computational curvilinear grid was developed using a varying grid resolution. The grid resolution ranged from $250 \mathrm{~m}$ at the offshore eastern boundary, where depths vary between $70 \mathrm{~m}$ to $80 \mathrm{~m}$ and increased towards the study area to ensure a resolution of no greater than $10 \mathrm{~m}$ in the nearshore region of Barrier No.2.

The wave model was calibrated over eight storm events during which a nearshore wave buoy was deployed approximately $100 \mathrm{~m}$ east of Barrier No. 2. Over 27 separate model setups were assessed, with each setup containing a unique permeation of bed friction, wave breaker and white capping parameters. The calibrated model has resulted in a mean error of $0.23 \mathrm{~m}$ and a Root-Mean-Square (RMS) error of $0.29 \mathrm{~m}$.

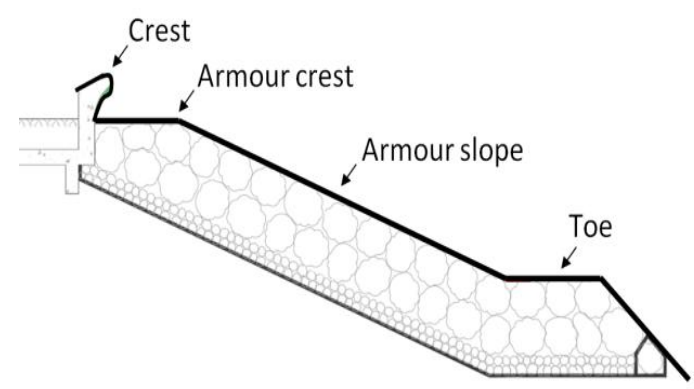

Fig. 2. Key components of coastal defense schematisation in the Neural Network.

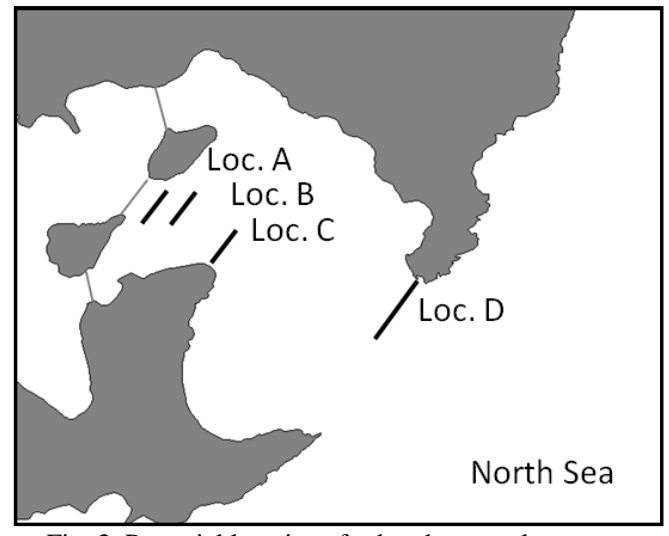

Fig. 3. Potential locations for breakwater placement

Wave overtopping modelling has been undertaken using the Neural Network (NN) calculation tool developed with the industry standard EurOtop Manual [2]. The NN tool provides the most suitable methodology to assess composite defences, which include seawall and armourstone breakwaters. The NN requires detailed geometric information on 15 of the defence characteristics, such as crest height, armour height, armour width, berm elevation, berm width, upper slope, lower slope and roughness. These have been schematised based on topographic survey of the barriers. Key components of a typical schematisation are shown in Fig. 2. The NN was calibrated using the nearshore SWAN results during a monitoring period where two coastal storms caused waves to overtop Barrier No. 2. These conditions were replicated within the $\mathrm{NN}$ using a roughness coefficient of 0.55 to represent the current armourstone. A series of design storm events were subsequently run through the SWAN and the NN models, which indicated the barrier has a current standard of defence of 1 in 1-years (i.e. overtopping occurs annually), which matches anecdotal records.

The wave model was used to assess four possible breakwater locations (Fig. 3) to improve the standard of defence to under a $10 \mathrm{~L} / \mathrm{s} / \mathrm{m}$ overtopping rate during a 1 in 200-year event. Location A considered a nearshore detached breakwater, Location B considered a breakwater that could be constructed using a temporary access road, and Locations $\mathrm{C}$ and $\mathrm{D}$ considered breakwaters attached to the shoreline where a land-based construction approach could be used.

Each breakwater was represented in the SWAN wave model and the nearshore wave height calculated adjacent to Barrier No. 2. The amount of overtopping was then calculated using the NN tool. The length of each breakwater was varied to limit overtopping to $10 \mathrm{~L} / \mathrm{s} / \mathrm{m}$ during a 1 in 200-year joint probability storm event. Each breakwater was assessed in terms of its required length, depth and volumetric requirements (i.e. required armour). Preliminary assessments indicate that a $400 \mathrm{~m}$ breakwater at Location B would achieve the design targets, as shown Fig. 4.

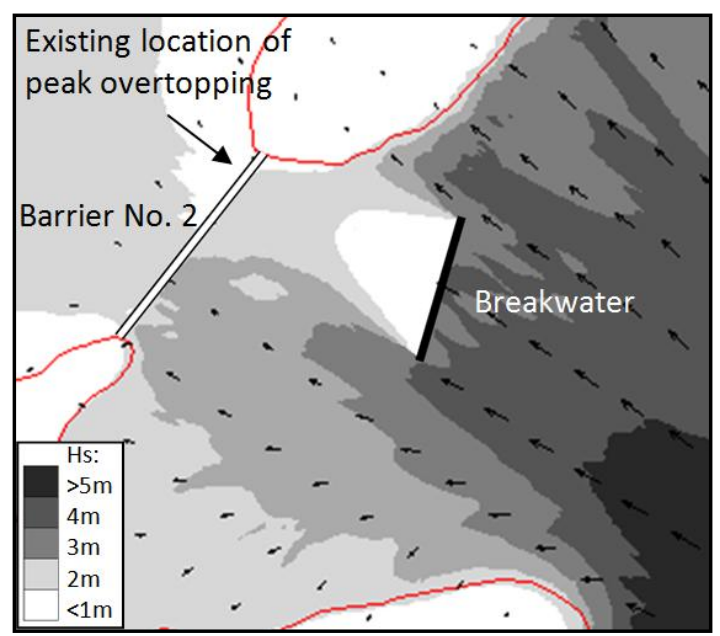

Fig. 4. Nearshore wave patterns for a $200 \mathrm{~m}$ breakwater at Location B.

\section{DESIGN OF ARMOURSTONE}

Once placement was optimised using the SWAN model the preliminary structural design was undertaken to ensure stability during 1 in 200-year conditions over a lifetime of 100 years. Over this period the structure has a $39 \%$ chance of encountering a 200-year event, and therefore calculations involving extreme conditions were required to be robust. Several successive calculations were undertaken to develop the conceptual breakwater. These have included the calculation of primary armour size, secondary armour size, crest elevation, crest width, toe protection, trunk and head design and selection of construction methods. Armour has been sized using both the Hudson (1974) and Van Der Meer (1988) equations, with the latter described within this paper. Of most importance to coastal engineering design is the calculation of the armourstone size able to withstand extreme conditions for the design life. Empirical calculations such as the Van Der Meer equation are widely used, which relates a stable armour size (or weight) to the design wave conditions:

$$
D_{n}=\frac{H_{S}}{6.2 . S^{0.2} P^{0.18} N_{z}^{-0.1} \varepsilon_{m}^{-0.5} \Delta}
$$


where, $D_{n}$ is the nominal median diameter of armour blocks $(m), \mathrm{H}_{\mathrm{s}}$ is the design wave height, $S$ is the nominal damage, $P$ is the nominal permeability, $N_{z}$ is the number of waves, $\varepsilon$ is the surf similarity parameter and $\Delta$ is the dimensionless relative buoyant density of rock. The formulation of the Van Der Meer equation places an emphasis on the wave conditions at the toe of the structure. It is the estimation of these wave conditions that have the most uncertainty, therefore introducing a level of risk into the design process. Numerical modelling is not free of these uncertainties, which are inherent in many aspects of the modelling process. These include the use of collected data (instrument response, statistical sampling density), use of offshore boundary conditions (extreme data analysis methods, need for extrapolation) and the modelling approach (type of model and numerical solution). Additionally the increase in future sea levels and wave conditions due to climate change must be considered, which incorporates additional uncertainty into the design process.

Three standard methods have been initially used to account for the inherent uncertainty within armour calculations to manage the design risk. These are:

1) The use of deterministic design formulae using a range of joint probability extreme wave, sea level and climate change events.

2) The use of an ultimate limit state, based on the improbable scenario of a 200-year wave condition, 200-year extreme sea-level and 95 percentile climate change estimate occurring simultaneously.

3) The use a probabilistic design approach by incorporating partial safety factors as described within the USACE CEM.

\section{A. Extreme Joint Probability}

Approach A has used a joint probability analysis to determine the most scientifically 'realistic' combination of water level and wave conditions that constitutes the worst case scenario for a 200-year return period. For any return period there are several combinations of waves and sea levels which are expected to be exceeded once within the given period. For example, for a 10 -year joint return period with for a high level of dependence, the combinations might include a 1 -year wave with a 10 -year sea level, a 10 -year wave with a 1-year sea level, or combinations in between.

The joint probability assessment has been undertaken using the established Defra methodology [3] considered best practise in the UK, and assessed against the level of dependence between surge and wave conditions in the Orkney Islands. The analysis has resulted in ten potential scenarios for the 1 in 200-year return period. Additionally, an estimate of potential climate change has been added to each scenario based on the UK Climate Projections (UKCP09) [4] to the year 2115. Within UKCP09 estimates for sea level rise are provided under low, medium and high emissions scenarios, which are further refined by 5th, 50th and 95th percentile confidence ratings. In simple terms this should be interpreted as the relative likelihood of the predicted change being at, or less than, the given change. For the conceptual design the medium emissions scenario was considered using the 95th percentile confidence rating, which gave a projected sea level rise of $0.72 \mathrm{~m}$ for the year 2115 at the study site.
Each of the joint probability 200-year plus climate change scenarios are presented in Table I.

TABLE I: OFFSHORE 1 IN 200-YEAR JOINT PROBABILITY SCENARIOS

\begin{tabular}{ccc}
\hline $\begin{array}{c}\text { Water level } \\
(\mathrm{mCD})\end{array}$ & $\begin{array}{c}\text { Wave height } \\
(\mathrm{m})\end{array}$ & Wave period $(\mathrm{s})$ \\
\hline 2.61 & 7.53 & 13.24 \\
2.70 & 6.95 & 12.53 \\
2.97 & 6.73 & 12.26 \\
2.96 & 6.73 & 12.26 \\
3.06 & 6.10 & 11.36 \\
3.23 & 5.80 & 11.12 \\
3.31 & 5.51 & 10.09 \\
3.42 & 5.11 & 9.27 \\
3.49 & 4.82 & 8.99 \\
3.57 & 4.52 & 8.85 \\
\hline \hline
\end{tabular}

Each joint probability event was simulated using the SWAN model with the nearshore wave conditions extracted at the toe of the proposed breakwater. The significant wave height ranged between 3.5 and $4.0 \mathrm{~m}$.

Using the Van Der Meer equation the required armor size and weight has been assessed for each 1 in 200-year joint probability scenario for a 1 in 4 slope, resulting in a worst-case stable armour diameter of $1.5 \mathrm{~m}$, or 9.3 tonnes.

\section{B. Ultimate Limit State}

While Approach A has utilised a range of potential 1 in 200-year scenarios, there remains uncertainty in the degree of correlation between extreme wave and water level conditions. If this correlation is under-predicted, it is likely that the breakwater will experience an event beyond its design conditions. In order to incorporating a conservative factor of safety into the design process Approach B has developed an Ultimate Limit State (ULS) by producing an event with an extremely low probability. The ULS has been based on a design scenario with a 1 in 200-year offshore wave event combined with 1 in 200-year extreme sea level, and incorporates the 95th percentile, medium emissions sea-level rise scenario.

The ULS scenario was simulated using the SWAN model and the nearshore wave conditions extracted at the toe of the proposed breakwater. The extreme significant wave height was calculated to be $4.6 \mathrm{~m}$. Using the Van Der Meer equation the required armour size and weight has been calculated to be approximately $1.7 \mathrm{~m}$, or 13.0 tonnes.

\section{Partial Safety Factors}

Approach A and B have been based on the best available extreme conditions and climate change estimates. However, there remains additional uncertainty within the offshore extreme conditions, climate change estimates and modelling accuracy that are not reflected within these calculations.

Approach $\mathrm{C}$ has used a probabilistic design method to account for higher levels of uncertainty. The use of partial safety factors (PSF) is described within a number of design manuals including the CEM and the Eurocodes, where key design parameters have been assigned individual factors of safety. Two PSF can be used in design formula, one to account for uncertainty in the nearshore wave parameters $\left(\gamma_{S}\right)$ and one to account for uncertainties within the armourstone calculations $\left(\gamma_{R}\right)$. Large values, e.g. $\gamma_{S}=1.7$, indicates a 
relatively large sensitivity of wave parameters, while values approaching 1 indicates little or negligible sensitivity.

Applying a probabilistic design the Van Der Meer equation (Equation 1) has been rewritten as:

$$
\frac{6.2 . S^{0.2} P^{0.18} N_{Z}^{-0.1} \varepsilon_{m}^{-0.5} \Delta D_{n}}{\gamma_{R}}-\gamma_{S} H_{d e s}=0
$$

where the $\gamma_{R}$ allows for a reduction to the armourstone resistance and $\gamma_{S}$ allows for additional wave forces. Standard PSF have been adopted from the CEM based on published information on the extreme wave properties used within the numerical modelling. The nearshore wave conditions calculated within the ten joint probability scenarios described in Approach A have been combined with a PSF that assumes a good knowledge of wave properties, and has a normal (Gaussian) distribution with a standard deviation of 0.05.

The modified Van Der Meer equation was used to calculate the required armour size and weight for 1 in 4 slope. The worst case calculations resulted in a stable armour size of $2.0 \mathrm{~m}$, or 20.5 tonnes.

\section{VAlidation Through a Monte CARlo Approach}

The range of armourstone sizes calculated through each of the above methods demonstrates the engineering judgment required in coastal engineering. In particular, the manner in which uncertainty within wave, sea level and climate change estimates are accounted for within the design process can have a large effect on structural requirements.

Ensemble wave modelling was undertaken using a simplified Monte Carlo approach to further refine the armourstone calculations. In order to undertake this assessment the uncertainty in storm driving variables was investigated. Extreme offshore wave estimates and water levels have been sourced from the latest UK Environment Agency estimates [5]. The data used to make these estimates is limited to less than 100-years of records; therefore there is a degree of uncertainty associated with the values. The confidence level is considered to be $0.5 \mathrm{~m}$ for the 1 in 200 -year extreme sea level estimate and $1.0 \mathrm{~m}$ for extreme offshore wave estimate at the study site. All future sea-levels have been based on the medium emissions climate change scenario, which is $0.14 \mathrm{~m}$ lower than the high emissions scenario at the study site. There is little confidence in the expected changes to wave height due to climate change, with UKCP09 stating a potential increase of $1.0 \mathrm{~m}$ and decrease of $1.5 \mathrm{~m}$.

Monte Carlo simulations were carried out for the Joint Probability Run No 3 (refer to Table I) which resulted in the largest stable rock size using Approach A. The scenario was run using 500 new simulations which incorporated varying levels of uncertainty based on the limits identified above. Several assumptions were made in order to generate the Monte Carlo simulations. The confidence limits have been considered to encapsulate $95 \%$ of the uncertainty, considered to be two standard deviations from the mean under a Gaussian probability distribution (where the mean is considered to be the published 200-year extreme sea-level and wave estimates). The uncertainty within each parameter was considered to be independent of other parameters.
Simulations were developed to account for varying degrees of extreme wave uncertainty $( \pm 1 \mathrm{~m})$, extreme water level uncertainty $( \pm 0.5 \mathrm{~m})$, changes to climate change emissions scenarios $( \pm 0.14 \mathrm{~m})$ and potential changes to wave height $( \pm 1.0 \mathrm{~m})$.

The nearshore wave conditions were extracted from the SWAN model and used to calculate the range of required armourstone, as shown in Fig. 5. The figure shows an approximately normal distribution ranging between 5 and 13 tonnes. Also shown on the figure are the initial armour estimates based on Approaches A, B and C. As the basis for the simulations, Approach A accounts for approximately half the modelled uncertainty. The ULS within Approach B accounts for all of the simulated uncertainty, and the use of PSF within Approach $\mathrm{C}$ far exceeds all simulations. Using the Monte Carlo approach the required armour size has been investigated to account for a specific proportion of the uncertainty, as shown in Table II. In order to account for $95 \%$ of the modelled uncertainty an armour weight of 11.5 tonnes would be required.

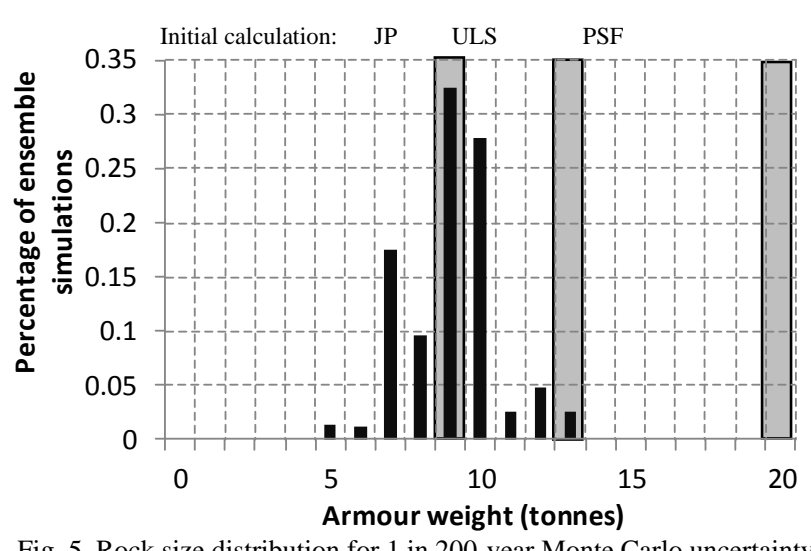

Fig. 5. Rock size distribution for 1 in 200-year Monte Carlo uncertainty simulations.

TABLE II: REQUIRED ARMOURSTONE MASS TO EXCEED SIMULATED WAVE, SEA-LEVEL AND ClimATE CHANGE UNCERTAINTY

\begin{tabular}{cc}
\hline \hline $\begin{array}{c}\text { Armour mass } \\
\text { (tonnes) }\end{array}$ & $\begin{array}{c}\text { Percentage } \\
\text { exceedance }(\%)\end{array}$ \\
\hline 9.5 & $75 \%$ \\
9.6 & $80 \%$ \\
9.8 & $85 \%$ \\
10.1 & $90 \%$ \\
11.5 & $95 \%$ \\
12.9 & $100 \%$ \\
\hline \hline
\end{tabular}

\section{RESULTS AND DISCUSSION}

A rubble mound breakwater has been considered to address wave overtopping experienced at the Churchill Barrier No. 2, in the Orkney Islands, UK. The conceptual design of the proposed breakwater has been optimised by numerical modelling software, using the SWAN wave model and the Neural Network wave overtopping tool to limit overtopping to $10 \mathrm{~L} / \mathrm{s} / \mathrm{m}$ during a 1 in 200-year joint probability storm event.

The breakwater has been designed to structurally withstand a 1 in 200-year event over a design life of 100 years. Over this period the structure has a $39 \%$ chance of encountering such an event, and therefore calculations involving extreme conditions were required to be robust. 
Three methods have been investigated to account for the inherent uncertainty within armourstone calculations and to mitigate the risk of undersizing rock armour.

The results of the three armourstone calculations shows the variability between approaches to manage uncertainty, and provides an upper limit of armour weight for the conceptual design. The use of the deterministic Van Der Meer (1988) formula for a range of 200-year joint probability extreme scenarios resulted in the lowest stable armourstone weight of 9 tonnes. The ultimate limit state considered additional uncertainty by producing an event with an extremely low probability. The approach resulted in an increased stable armour weight of 13 tonnes. Lastly, while not used widely within the UK, a probabilistic design approach was considered to account for the remaining uncertainty at the site. The approach resulted in an increased stable armour weight of 20 tonnes.

To address this range of armourstone sizes, ensemble wave modelling was undertaken using a simplified Monte Carlo approach where 500 different simulations were developed accounting for various levels of uncertainty within extreme sea-level, wave and climate change estimates. In order to account for $95 \%$ of the modelled uncertainty an armour weight of 12 tonnes would be required.

\section{REFERENCES}

[1] U.S. Army Corps of Engineers, Coastal Engineering Manual, Engineer Manual 1110-2-1100, vol. 6, Washington, D.C., 2002.

[2] T. A. Pullen, N. W. H. Bruce et al. European manual for the assessment of wave overtopping. [Online]. Available: http://www.overtopping-manual.com

[3] Use of Joint Probability Methods in Flood Management: A Guide to Best Practice, R\&D Technical Report FD2308/TR2,
Defra/Environment Agency Flood and Coastal Defence R\&D Programme, March 2005.

[4] UK Climate Change Projections 2009. [Online]. Available: http://ukclimateprojections.metoffice.gov.uk/

[5] UK Environment Agency, "Coastal flood boundary conditions for UK mainland and islands," Project: SC060064/TR2 and TR3: Design Swell-Waves and Design Sea-Levels, Environment Agency, Feb. 2011.

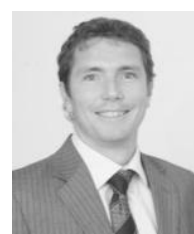

Daniel Rodger is a chartered senior coastal engineer with JBA Consulting, UK, specializing in coastal design and numerical modelling for large scale engineering, dredging, nourishment and coastal defence projects. He is a chartered member of the Institute of Marine Engineering, Science and Technology (IMarEST) and the UK engineering council and has a bachelor of environmental science with a major in marine science, a master of engineering, a post-graduate diploma in geographical information science and a post-graduate certificate in coastal engineering.

Dan has 9 years international experience in Australia, United Kingdom, Ireland and France. He has worked in both private and public sectors, initially within Local Government managing dredging, beach nourishment and estuary/coastal management works, and later as an engineering consultant advising on coastal engineering works and numerical modelling. $\mathrm{He}$ is currently the team leader for Coastal Processes within JBA's Coastal Risk Management Group, where he manages a team undertaking detailed hydrodynamic, wave and sediment transport modelling and design of coastal structures.

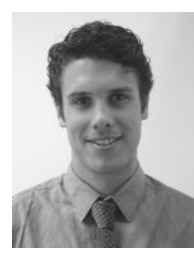

Josh Harris is a coastal engineer with JBA Consulting, UK and a graduate member of the Institution of Civi Engineers. He has a background in coastal processes and engineering having completed a master in coastal engineering at the University of Plymouth. Josh has experience working on coastal design and modelling projects involving wave transformation, overtopping, inundation and forecast modelling. Josh is involved in the development of SWAN wave transformation models to calculate near shore wave conditions for use in overtopping and breakwater design projects for both the public and private sectors. 